\title{
A Nurse-Led Telephone Session and Quality of Life after Radiotherapy Among Women with Breast Cancer: A Randomized Trial
}

\author{
Birgit Bjerre Høyer ${ }^{*}, 1,2$, Gunnar Vase Toft ${ }^{1}$, Jeanne Debess ${ }^{3}$ and Cecilia Høst Ramlau-Hansen ${ }^{1,4}$ \\ ${ }^{I}$ Department of Occupational Medicine, Aarhus University Hospital, Aarhus, Denmark \\ ${ }^{2}$ Department of Oncology, Vejle Hospital, Vejle, Denmark \\ ${ }^{3}$ University College of Northern Denmark, Aalborg, Denmark \\ ${ }^{4}$ Department of Epidemiology, Institute of Public Health, University of Aarhus, Aarhus, Denmark
}

\begin{abstract}
The purpose of this study was to investigate whether a nurse-led telephone session with patients suffering from breast cancer approximately ten days after final radiotherapy treatment affected their quality of life two to four weeks after radiotherapy. The study was conducted at the Radiotherapy ward at Vejle Hospital, Denmark between January and May 2010. The study population consisted of 100 patients, who were randomized with a 1:1 ratio to have either ordinary supportive conversations (control group), or ordinary supportive conversations and a supplementary nurse-led telephone session (intervention group). The quality of life was assessed using the questionnaires EORTC QLQ-C30 and EORTC QLQ-BR23. For statistical comparison of quality of life and for adjustment for covariates, multiple linear regression analysis was conducted. The mean [95 \% CI] quality of life was 72.0 [66.4-77.6] in the control group and 69.9 [64.3-75.2] in the intervention group. Adjustment for covariates did not change the estimates. No statistically significant differences were found in the groups in either of the analyses. The nurse-led telephone session had no positive effect on the quality of life of patients with breast cancer two to four weeks after their final radiotherapy.
\end{abstract}

Keywords: Quality of life, breast neoplasm, questionnaire, RCT, supportive conversation, nursing, women.

\section{INTRODUCTION}

Breast cancer is the most frequent type of cancer in Scandinavia, excluding skin cancer [1]. In 2008, the age standardized incidence rate of breast cancer in Scandinavia was $88.6 / 100,000$ person years and the prevalence rate was $1764 / 100,000$ person years [2]. The main treatment for breast cancer is surgery followed by chemotherapy, radiotherapy, anti-hormones and/ or antibody treatment.

In Denmark, radiotherapy is ordinarily given daily five days a week for five to six weeks [3]. The treated women experience varying degrees of side effects of radiotherapy, such as skin burns, dyspnoea and malaise [4]. These side effects peak 10 to 14 days after the end of the treatment. Normally, the patients are not seen in the hospitals until six to 12 weeks after the end of the radiotherapy. American studies indicate that breast cancer patients feel vulnerable and insecure and express a need for follow-up, as they feel abandoned and have a poor quality of life $[5,6]$.

Previous studies of quality of life among women with breast cancer have mainly focused on extended conversations and teaching sessions conducted by psychologists $[7,8]$. To minimize costs, telephone sessions have been conducted $[9,10]$. In a recent randomized study, Salonen et al. found no effect of a telephone session one week after surgery on quality of life, but the intervention

*Address correspondence to this author at the Department of Occupational Medicine, Aarhus University Hospital, Nørrebrogade 44, Build. 2C, 8000 Aarhus C, Denmark; Tel: +45 894942 75; Fax: +45 894942 60;

E-mail: birghoey@rm.dk group had improvements concerning body image, future perspectives and side effects after surgery [11]. McArdle et al. found that a nurse-led telephone session induced decreased morbidity, e.g. less anxiety, insomnia and social dysfunction [12]. Interventions which had a total of less than 20 hours of conversation had the greatest effect on quality of life, anxiety and depression according to a systematic review by Naaman et al. [13]. However, not all agree upon the efficiency of nurse-led interventions for women suffering from breast cancer. Wengstrom et al. found no effect of seven nurse sessions at 30 minutes each concerning quality of life in general and side effects, but they found that the intervention group had a reduced stress level compared to the control group [14].

Efficace et al. reported that baseline quality of life had an effect on survival in women with metastatic breast cancer [15], but others found diverging results $[16,17]$. Debess et al. observed that women with breast cancer who had just finished their anti-hormone treatment or chemotherapy had a poorer quality of life compared to the control group of healthy women [18]. In the case of good quality of life enhancing life for severely ill people, there is a need for studies that improve the quality of life of women with breast cancer.

The present study aims to investigate whether one telephone session approximately ten days after the final radiotherapy treatment, with a nurse from the radiotherapy ward, where the information from the last session is repeated, affects the global quality of life for women with newly diagnosed primary breast cancer two to four weeks 
after final radiotherapy treatment compared to the ordinary procedure without telephone sessions.

\section{MATERIALS AND METHODS}

\section{Study Design}

This was a single-center, parallel group study with balanced randomization (1:1) conducted in Denmark.

\section{Participants and Settings}

Eligible participants were women 18 years or more with newly diagnosed primary breast cancer referred to the oncology ward at Vejle Hospital, Denmark for radiotherapy treatment, who had had either a lumpectomy or mastectomy, had access to a telephone, had sufficient hearing and spoke Danish. Exclusion criteria were prior breast cancer, prior or current serious illness, e.g. embolus or other cancers, or prior or current depression, dementia, anxiety or other psychiatric illness. One hundred patients were included in the study between January and May 2010.

\section{General Nursing Sessions for All Included Participants}

All patients with breast cancer had either one or two sessions prior to and during radiotherapy treatment. Prior to treatment, a doctor and a nurse informed them of the effects and side effects of the performed surgery, former or future chemotherapy, anti-hormones, and the impending radiotherapy. Shortly before the end of the radiotherapy, all patients had a final session with a nurse relating to the radiotherapy period, side effects, treatment of side effects, medication during and after radiotherapy, mental state of mind, social conditions and prospective check-ups six to twelve weeks after radiotherapy.

\section{Intervention}

Patients were randomly assigned either to receive a telephone session 10 days after the final radiotherapy or to have no contact with the Department of Oncology before the usual check-up six to 12 weeks after radiotherapy. The intervention was a telephone session which was conducted according to the same template as the last session prior to the final treatment. The nurse made sure to cover all the topics described above. The intervention was carried out by four experienced nurses who had between six and 20 years of oncology experience. The duration of the telephone sessions was 10 to 30 minutes.

\section{Outcome}

The primary outcome concerned global quality of life. Secondary outcomes were side effects and specific symptoms. All patients included in the study rated the outcomes on the Danish version of the questionnaires EORTC QLQ-C30 and EORTC QLQ-BR23 two to four weeks after the final radiotherapy. This was four to 18 days after the telephone session in the intervention group. The questionnaires are developed by the European Organization for Research and Treatment of Cancer (EORTC), which is a group of European oncologists and researchers who have worked with cancer research since 1962. EORTC QLQ-C30 is the global, cancer-specific questionnaire which is used to examine health-related quality of life among patients with cancer. The questionnaire was developed in 1993 and has been validated and revised several times [19]. The questionnaire is divided into five functional scales (physical, role, emotional, cognitive and social functioning) and nine symptom scales and single items (fatigue, nausea/vomiting, pain, dyspnoea, insomnia, appetite loss, constipation, diarrhea and financial difficulties). Side effects and specific symptoms among breast cancer patients were measured using EORTC QLQ-BR23 which is a supplement to EORTC QLQ-C30 developed in 1996. The questionnaire measures symptoms and side effects specifically among breast cancer patients [19]. The questionnaire is divided into five multiitem scales and examines side effects, arm symptoms, breast symptoms, body image and sexual functioning. Furthermore, the questionnaire examines sexual pleasure, hair loss and future perspectives using single items [19].

The responses were analyzed using the domains outlined in the scoring manual [20]. All scales and single items consisted of scales of either one to four or one to seven, and they were transformed into scores from one to 100. A high score meant a high response level; correspondingly, a high score in the functional scales meant a high level of function, a high score in global health meant a high self-rated health and quality of life, and high scores in the symptom scales meant a high level of symptoms [20].

\section{Sample Size}

No similar study has found a difference in quality of life. To justify the study we wanted to detect a difference in quality of life of 10-15 percent points. To detect a difference in quality of life of 10.5 points (SD 18.5) with a two-sided 5 $\%$ significance level and a power of $80 \%$, a sample of 49 patients in each group was necessary. We set the study sample to 100 to allow for loss to follow-up. To recruit 100 patients a three month period was anticipated.

\section{Randomization}

For allocation of the participants, a computer-generated list of random numbers was used. A randomization sequence was created using the website www.random.org with a 1:1 allocation using random block sizes of 10 . The nurse who held the session with the patients at the end of the radiotherapy treatment performed the randomization. She was unaware of which intervention group the patient was allocated to. The patients were unaware of which group they were assigned to until they received either the telephone call or the questionnaire measuring quality of life. An investigator who was not otherwise involved in the project kept a log of the groups.

\section{Blinding}

Patients became aware of which group they had been allocated to only at the time of the telephone call or when they received the questionnaire, whereas the data analyst was kept blinded to the allocation throughout.

\section{Statistical Analyses}

Baseline characteristics of the two groups were compared by chi squared test for categorical data and unpaired t-test for continuous data. Quality of life data are presented as mean with $95 \%$ confidence intervals $(95 \% \mathrm{Cl})$. A multiple linear regression was used to compare groups. Intention-to-treat analysis was the primary analysis. A per protocol analysis was 


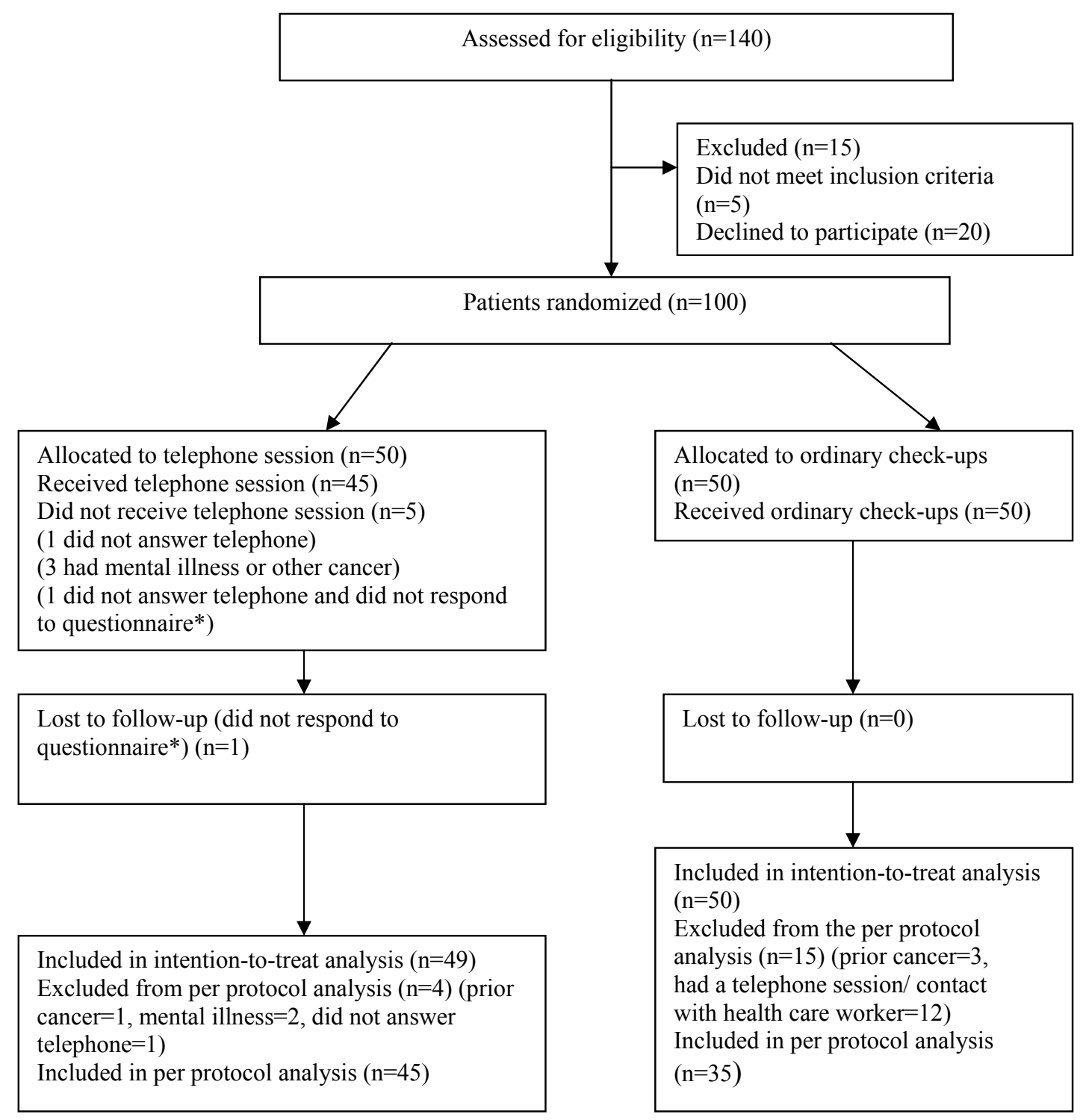

Fig. (1). Flow diagram of a single center trial of nurse-led telephone session $v$ s ordinary check-ups. The diagram includes detailed information on the excluded participants. * the same patient.

performed to assess whether protocol violation had influenced the result. In the per protocol analysis, any patients who should not have been included in the study, due to the inclusion criteria, were excluded. In the control group we excluded patients who had a telephone session or other types of sessions with health care providers. In the intervention group, we excluded patients who did not have a telephone session.

To determine whether a missing response had influenced the result, a sensitivity test was performed. A priori we decided that "type of operation", "anti-hormone treatment" and "treatment" could be possible confounders and thus a multiple linear regression was conducted. Statistical differences with a $p$ value $\leq 0.05$ were considered statistically significant. Data were analyzed using Stata ${ }^{\mathrm{TM}}$ 11.1.

\section{Ethics}

All patients signed an informed consent form prior to participation. The study was authorized by the Danish Data Protection Agency, journal number 2009-41-3937.

\section{RESULTS}

A total of 140 patients with breast cancer visited the radiotherapy ward during the study period. Fifteen patients were excluded due to the exclusion criteria, five patients did not fulfill the inclusion criteria and 20 patients did not want to participate for various reasons. In total, 100 patients were included, 50 in the intervention group and 50 in the control group. One patient in the intervention group was lost to follow-up due to failure to respond. The intervention group and the control group were quite homogeneous, but it was statistically significant that more women in the intervention group had a combination treatment in comparison with the control group (indicated by the variable "treatment") and statistically significant that more women in the intervention group had anti-hormone treatment in comparison with the control group (indicated by the variable "anti-hormone treatment"). Most women had had a lumpectomy, had a short or medium long education and were married or cohabiting. 
Mean age (SD) in the intervention group was 59 (9) years, and 61 (8) years in the control group (Table 1).

The primary analysis was intention-to-treat and included all patients who were randomly assigned and not lost to follow-up. Four patients in the intervention group and fifteen patients in the control group were protocol violators and thus 80 patients were included in the per protocol analysis.

The mean $(95 \% \mathrm{CI})$ score of quality of life was 69.9 $(64.3 ; 75.2)$ in the intervention group and $72.0(66.4 ; 77.6)$ in the control group. There was no statistically significant difference in quality of life between the groups. There was no statistically significant difference between the groups in the additional scales and items. Insomnia was borderline statistically significantly different between groups $(p=0.06)$. The point estimates for insomnia were mean $(95 \% \mathrm{CI}) 32.0$ $(23.0 ; 41.0)$ in the intervention group and $20.0(11.1 ; 28.9)$ in the control group (Table 2). The point estimates of constipation were mean $15.6[9.6 ; 21.7]$ in the intervention group and $7.5[1.5 ; 13.5]$ in the control group and was close to being statistically significantly different.

There were no statistically significant differences between the groups concerning side effects and symptoms. However, the point estimates for breast symptoms differed considerably. In the intervention group, the breast symptoms were mean $40.5(34.4 ; 46.6)$ and in the control group, the breast symptoms were mean $34.6(28.7 ; 40.6)$ (Table 3$)$.

As a sub-analysis, a per protocol analysis was conducted. In this analysis, patients who were included by mistake and patients who did not receive the intended intervention were excluded. The estimates do not change in the per protocol analysis in comparison to the intention-to-treat analysis. The quality of life was mean $70.6(64.6 ; 76.6)$ in the intervention group and $71.9(65.1 ; 78.7)$ in the control group (Table 4).

Table 1. Clinical and Demographic Characteristics of the Study Population

\begin{tabular}{|c|c|c|c|c|c|}
\hline \multirow{3}{*}{ Age Mean (SD) } & \multicolumn{2}{|c|}{ Intervention Group $(\mathbf{n}=\mathbf{5 0})$} & \multicolumn{2}{|c|}{ Control Group $(n=50)$} & \multirow{2}{*}{$\frac{\mathbf{P}}{0.22^{*}}$} \\
\hline & \multicolumn{2}{|c|}{$59(9)$} & \multicolumn{2}{|c|}{$61(8)$} & \\
\hline & $\mathbf{n}=$ & $(\%)$ & $\mathbf{n}=$ & $(\%)$ & \\
\hline \multicolumn{6}{|l|}{ Tumor stage $^{1}$} \\
\hline $\mathrm{T} 1$ & 27 & (54) & 24 & (48) & $0.18^{\wedge}$ \\
\hline $\mathrm{T} 2$ & 8 & (16) & 8 & (16) & \\
\hline $\mathrm{T} 3$ & 0 & $(0)$ & 1 & $(2)$ & \\
\hline Tis & 3 & (6) & 10 & (20) & \\
\hline Unknown & 12 & (24) & 7 & (14) & \\
\hline \multicolumn{6}{|l|}{ Operation } \\
\hline Lumpectomy & 40 & $(80)$ & 43 & (86) & $0.51^{\wedge}$ \\
\hline Mastectomy & 9 & (18) & 7 & (14) & \\
\hline Bilateral $^{2}$ & 1 & (2) & 0 & $(0)$ & \\
\hline \multicolumn{6}{|l|}{ Chemotherapy } \\
\hline Yes & 21 & (42) & 15 & (30) & $0.34^{\wedge}$ \\
\hline No & 27 & (54) & 34 & $(68)$ & \\
\hline Unknown & 2 & (4) & 1 & (2) & \\
\hline \multicolumn{6}{|l|}{ Anti-hormone therapy } \\
\hline Yes & 31 & $(62)$ & 18 & (36) & $0.02^{\wedge}$ \\
\hline No & 18 & (36) & 32 & (64) & \\
\hline Unknown & 1 & (2) & 0 & $(0)$ & \\
\hline \multicolumn{6}{|l|}{ Treatment } \\
\hline Radiotherapy only & 14 & (28) & 25 & $(50)$ & $0.04^{\wedge}$ \\
\hline Combination of treatments ${ }^{3}$ & 36 & (72) & 25 & $(50)$ & \\
\hline \multicolumn{6}{|l|}{ Civil status ${ }^{\#}$} \\
\hline Married/cohabiting & 42 & (86) & 38 & (76) & $0.22^{\wedge}$ \\
\hline Single/widow & 7 & (14) & 12 & (24) & \\
\hline \multicolumn{6}{|l|}{ Education $^{\#}$} \\
\hline Primary school/High school & 15 & (31) & 12 & (24) & $0.36^{\wedge}$ \\
\hline Blue collar/College & 32 & $(65)$ & 32 & (64) & \\
\hline University & 1 & (2) & 4 & $(8)$ & \\
\hline Unknown & 1 & (2) & 2 & (4) & \\
\hline
\end{tabular}

${ }^{\#}$ One missing patient in the intervention group. ${ }^{*} \mathrm{P}$-value derived from an un-paired t-test. ${ }^{\wedge} \mathrm{P}$-value derived from a chi squared test.

${ }^{\mathrm{I}}$ Tumor classification according to AJCC Cancer Staging Manual [21].

${ }^{2}$ Bilateral= double-sided, here meaning that the patient had had a lumpectomy on one side and a mastectomy on the other side

${ }^{3} \mathrm{~A}$ combination of radiotherapy and chemotherapy and/or anti-hormone therapy. 
Table 2. Quality of Life, Function and Symptom Scores from EORTC QLQ-C30 for the 99 Women who Responded to the Questionnaire. Intention-to-Treat Analysis

\begin{tabular}{|c|c|c|c|c|c|}
\hline \multirow{2}{*}{ QLQ-C30 Sub-Scales } & \multicolumn{2}{|c|}{ Intervention Group $(n=49)$} & \multicolumn{2}{|c|}{ Control Group $(n=50)$} & \multirow{2}{*}{$\mathbf{P}$} \\
\hline & $\mathbf{M}$ & $95 \% \mathrm{CI}$ & $\mathbf{M}$ & $95 \%$ CI & \\
\hline Global health status \& quality of life & 69.9 & $64.3 ; 75.2$ & 72.0 & $66.4 ; 77.6$ & 0.60 \\
\hline Physical functioning & 84.6 & $80.0 ; 89.2$ & 86.0 & $81.5 ; 90.6$ & 0.67 \\
\hline Role functioning & 75.5 & $68.4 ; 82.6$ & 77.7 & $70.6 ; 84.7$ & 0.67 \\
\hline Emotional functioning & 79.4 & $73.6 ; 85.3$ & 80.8 & $75.0 ; 86.6$ & 0.73 \\
\hline Cognitive functioning & 83.7 & $77.8 ; 89.6$ & 85.3 & $79.5 ; 91.2$ & 0.40 \\
\hline Social functioning & 88.4 & $83.2 ; 93.6$ & 88.7 & $83.5 ; 93.8$ & 0.95 \\
\hline Fatigue & 35.3 & $28.2 ; 42.3$ & 31.3 & $24.4 ; 38.3$ & 0.43 \\
\hline Nausea and vomiting & 7.8 & $2.7 ; 12.9$ & 4.7 & $-0.4 ; 9.7$ & 0.38 \\
\hline Pain & 21.4 & $14.7 ; 28.2$ & 20.3 & $13.7 ; 27.0$ & 0.82 \\
\hline Dyspnea & 14.3 & $7.9 ; 20.7$ & 13.3 & $7.0 ; 19.6$ & 0.83 \\
\hline Insomnia & 32.0 & $23.0 ; 41.0$ & 20.0 & $11.1 ; 28.9$ & 0.06 \\
\hline Appetite loss & 10.9 & $4.9 ; 16.9$ & 7.3 & $1.4 ; 13.3$ & 0.41 \\
\hline Constipation $^{1}$ & 15.6 & $9.6 ; 21.7$ & 7.5 & $1.5 ; 13.5$ & 0.06 \\
\hline Diarrhea & 11.6 & $4.9 ; 18.3$ & 9.3 & $2.7 ; 15.0$ & 0.64 \\
\hline Financial difficulties & 4.8 & $-0.26 ; 9.8$ & 6.0 & $1.0 ; 11.0$ & 0.73 \\
\hline
\end{tabular}

$\mathrm{M}=$ mean. $95 \% \mathrm{CI}=95 \%$ confidence interval. $\mathrm{P}$ derived from multiple linear regression. ${ }^{1}$ One patient missing in the control group.

Table 3. Function and Symptom Scores from EORTC QLQ-BR23 for the 99 Women Who Responded to the Questionnaire. Intention-to-Treat Analysis

\begin{tabular}{|c|c|c|c|c|c|}
\hline \multirow{2}{*}{ QLQ- BR23 Subscales } & \multicolumn{2}{|c|}{ Intervention Group (n=49) } & \multicolumn{2}{|c|}{ Control Group $(n=50)$} & \multirow{2}{*}{$\mathbf{P}$} \\
\hline & $\mathbf{M}$ & $95 \% \mathrm{CI}$ & $\mathbf{M}$ & $95 \%$ CI & \\
\hline Body image $^{1)}$ & 80.6 & $74.6 ; 86.6$ & 81.0 & $75.0 ; 87.0$ & 0.95 \\
\hline Sexual functioning $^{2)}$ & 19.4 & $13.6 ; 25.3$ & 21.4 & $15.4 ; 27.3$ & 0.76 \\
\hline Sexual pleasure ${ }^{3)}$ & 53.3 & $42.4 ; 64.3$ & 62.1 & $51.6 ; 72.6$ & 0.28 \\
\hline Future perspective ${ }^{1)}$ & 57.8 & $49.3 ; 66.3$ & 59.2 & $50.7 ; 67.7$ & 0.71 \\
\hline Systematic therapy side effects & 16.6 & $12.8 ; 20.3$ & 16.3 & $12.6 ; 20.1$ & 0.63 \\
\hline Breast symptoms $^{4)}$ & 40.5 & $34.4 ; 46.6$ & 34.6 & $28.7 ; 40.6$ & 0.09 \\
\hline Arm symptoms ${ }^{5)}$ & 16.1 & $10.8 ; 21.4$ & 14.7 & $9.5 ; 19.8$ & 0.60 \\
\hline Upset by hair loss ${ }^{6}$ & 6.9 & $0.87 ; 13.0$ & 7.5 & $1.5 ; 13.5$ & 0.78 \\
\hline
\end{tabular}

$\mathrm{M}=$ mean. $95 \% \mathrm{CI}=95 \%$ confidence interval. $\mathrm{P}$ derived from multiple linear regression.

${ }^{1)} 1$ patient missing in the control group. ${ }^{2)} 1$ patient missing in the intervention group and 4 patients missing in the control group. ${ }^{3)}$ Responses from patients who had been sexually active within the last week $=20$ patients in the intervention group and 22 patients in the control group. ${ }^{4} 2$ missing patients in the intervention group and 1 missing patient in the control group. ${ }^{5)} 2$ missing patients in the intervention group. ${ }^{6} 1$ missing patient in each group.

Table 4. Global Health of the 80 Patients Who Had the Intended Intervention. Per Protocol Analysis

\begin{tabular}{|c|c|c|c|c|c|}
\hline \multirow{2}{*}{ QLQ-C30 } & \multirow{2}{*}{ Intervention Group (n=45) } & \multicolumn{2}{|c|}{ Control Group (n=35) } \\
\cline { 2 - 5 } & M & $\mathbf{9 5 \% ~ C I}$ & M & $\mathbf{9 5 \%}$ CI \\
\hline \hline Global health status \& quality of life & 70.6 & $64.6 ; 76.6$ & 71.9 & $65.1 ; 78.7$ & 0.77 \\
\hline
\end{tabular}

$\mathrm{M}=$ mean. $95 \% \mathrm{CI}=95 \%$ confidence interval. $\mathrm{P}$ derived using a linear regression.

Additionally, a multiple linear regression analysis was conducted, adjusting for the variables "treatment", "operation" and "anti-hormone treatment". The crude estimate of quality of life was mean $69.9(64.3 ; 75.5)$ and the adjusted mean was $71.5(63.1 ; 79.9)$ in the intervention group. In the control group, the crude mean (95\% CI) quality of life was $72.0(66.4 ; 77.6)$ and the adjusted mean was $73.2(66.2 ; 80.1)$. 
To assess whether one missing patient in the intervention group had influenced the result, a sensitivity analysis was performed on the poorest and the best possible response from the missing patient. The result of the sensitivity test indicated that the result was not influenced by the missing patient in the intervention group.

\section{DISCUSSION}

This clinically controlled, randomized study found that a nurse-led telephone session with women treated for newly diagnosed primary breast cancer approximately ten days after the final radiotherapy had no significant effect on global quality of life measured as global health on the QLQC30 scale two to four weeks after the final radiotherapy treatment. Furthermore, the telephone session had no effect on functional scales, symptom scales, multi-item scales or single-item scales measured on QLQ-BR23. The result might even suggest that the intervention may be harmful, as the control group had a better quality of life in comparison with the intervention group.

The intervention in this study was quite small which might have caused the lack of a statistically significant difference in quality of life between the groups. A study which matched this study concerning the intervention and duration of session performed by a physiotherapist, did not find a difference in quality of life either, but did identify an improved body image, fewer side effects and less concern about the future [11]. An earlier study of the quality of life among Danish women using the EORTC QLQ-C30 found a global health of mean (SD) 74.8 (23.8), which is a somewhat better result than we found in this study [22]. The better quality of life found in the study by Klee et al. is consistent with the fact that their study examined healthy women. The women in this study suffered more from fatigue, constipation, diarrhea and nausea but had fewer financial difficulties than the healthy women in the study by Klee et al., but otherwise the results are quite similar [22].

Within quality of life research, a theory concerning "response shift" (a change in the meaning of people's selfevaluation) during chronic or severe illness has been developed [23]. Osborne et al. have argued that chronically ill people attending self-management programs feel either a negative response shift (people feel worse than before the program), no response shift (people feel about the same as prior to the program) or a positive response shift (people feel better than before the program) [24]. It is possible that the telephone session left the majority of patients in the intervention group with a negative response shift which could then be the reason that the patients in the intervention group did not report a higher quality of life than the patients in the control group.

The timing of the measurement of quality of life might be of significance to the response, and it is possible that two weeks after the final treatment is not the time where the intervention causes a changed quality of life. A longer follow-up might give another result.

As the study is randomized, it is expected that all possible confounders are equally distributed in the intervention and control groups. A priori three covariates were identified as possible confounders. A multiple linear regression was conducted to adjust for potential confounding by these covariates. Crude and adjusted results did not differ notably, and thus confounding by these covariates was not likely.

The systematic review by Naaman et al. found that interventions with a total of less than 20 hours were moderately associated with better quality of life [13]. The intervention in this study was of approximately 10 to 30 minutes and showed neither effect on quality of life nor any other measured variable. As the results concerning insomnia, constipation and breast symptoms in this study approached statistical significance contradicting the hypothesis, it is possible that the telephone session with information on the side effects actually induced symptoms that the women would not have felt had they not had a telephone session.

A limitation to this study was the fact that we did not assess quality of life at baseline. We assumed the baseline quality of life to be the same in the intervention group and the control group. If the baseline quality of life differed in the two groups, it is possible that the intervention did enhance the quality of life in the intervention group, but it is not possible to assess this change due to the design of this study.

Some women with breast cancer also suffer from comorbidity. These women were not investigated in this study and the result cannot be generalized to women with comorbidities. On the other hand, these results can be generalized broadly to radiotherapy wards treating similar women, as the women examined here were not specially selected.

It is possible that a change in the juncture of the intervention and the timing of the measurement of quality of life, as well as an intervention of a larger scale, would change the results. In the questionnaire, the women in the intervention group were asked to comment on the content and timing of the intervention. The majority of women were satisfied concerning both. A satisfaction survey might have found an association between the intervention and an improved degree of satisfaction. Coping is another outcome that might have found an association and further research needs to address these areas of interest.

\section{CONCLUSION}

The study found no statistically significant effect of the telephone session on quality of life and function and symptom scores measured two to four weeks after the final radiotherapy treatment. Despite the limited study size, the point estimates indicate that telephone sessions were not associated with an improved quality of life, but perhaps with the opposite.

\section{ACKNOWLEDGEMENTS}

The authors wish to thank Nurse Mette Giørtz for her invaluable help running the study in the Radiotherapy ward and including patients during the study period. We also want to thank the management and the staff at the Department of Oncology and Radiotherapy ward at Vejle Hospital for their cooperation making this study possible. This work was financially supported by Roche. 


\section{REFERENCES}

[1] Moller B, Fekjaer H, Hakulinen T, et al. Prediction of cancer incidence in the Nordic countries up to the year 2020. Eur J Cancer Prev 2002; 11(Suppl 1): S1-96.

[2] Engholm G, Ferlay J, Christensen N, et al. NORDCAN: Cancer Incidence, Mortality and Prevalence in the Nordic Countries, Version 3.4. Association of Nordic Cancer Registries. Danish Cancer Society. 2009. Available at: www.ancr.nu [Cited:10 December 2009].

[3] Overgaard M, Christensen JJ. Postoperative radiotherapy in DBCG during 30 years. Techniques, indications and clinical radiobiological experience. Acta Oncol 2008; 47(4): 639-53.

[4] Fiets WE, van Helvoirt RP, Nortier JW, van der Tweel I, Struikmans H. Acute toxicity of concurrent adjuvant radiotherapy and chemotherapy (CMF or $\mathrm{AC}$ ) in breast cancer patients: a prospective, comparative, non-randomised study. Eur J Cancer 2003; 39(8): 1081-8.

[5] Cappiello M, Cunningham RS, Knobf T, Erdos D. Breast cancer survivors: Information and support after treatment. Clin Nurs Res 2007; 16(4): 278-93.

[6] Ganz PA, Kwan L, Stanton AL, et al. Quality of life at the end of primary treatment of breast cancer: first results from the moving beyond cancer randomized trial. J Natl Cancer Inst 2004; 96(5): 376-87.

[7] Aranda S, Schofield P, Weih L, Milne D, Yates P, Faulkner R. Meeting the support and information needs of women with advanced breast cancer: a randomised controlled trial. Br J Cancer 2006; 95(6): 667-73.

[8] Arving C, Sjödén P, Bergh J, et al. Satisfaction, utilisation and perceived benefits of individual psychosocial support for breast cancer patients - A randomised study of nurse $v s$ psychologist interventions. Patient Educ Couns 2006; 62(2): 235-43.

[9] Coleman EA, Tulman L, Samarel N, et al. The effect of telephone social support and education on adaptation to breast cancer during the year following diagnosis. Oncol Nurs Forum 2005; 32(4): 8229.

[10] Marcus AC, Garrett KM, Cella D, et al. Telephone counseling of breast cancer patients after treatment: a description of a randomized clinical trial. Psychooncology 1998; 7(6): 470-82.

[11] Salonen P, Tarkka MT, Kellokumpu-Lehtinen PL, Astedt-Kurki P, Luukkaala T, Kaunonen M. Telephone intervention and quality of life in patients with breast cancer. Cancer Nurs 2009; 32(3): 17790.

[12] McArdle JM, George WD, McArdle CS, et al. Psychological support for patients undergoing breast cancer surgery: a randomised study. BMJ 1996; 312(7034): 813-6.
[13] Naaman SC, Radwan K, Fergusson D, Johnson S. Status of psychological trials in breast cancer patients: a report of three metaanalyses. Psychiatry 2009; 72(1): 50-69.

[14] Wengstrom Y, Haggmark C, Strander H, Forsberg C. Effects of a nursing intervention on subjective distress, side effects and quality of life of breast cancer patients receiving curative radiation therapy--a randomized study. Acta Oncol 1999; 38(6): 763-70.

[15] Efficace F, Biganzoli L, Piccart M, et al. Baseline health-related quality-of-life data as prognostic factors in a phase III multicentre study of women with metastatic breast cancer. Eur J Cancer 2004; 40(7): 1021-30.

[16] Efficace F, Therasse P, Piccart MJ, et al. Health-related quality of life parameters as prognostic factors in a nonmetastatic breast cancer population: an international multicenter study. J Clin Oncol 2004; 22(16): 3381-8.

[17] Luoma ML, Hakamies-Blomqvist L, Sjostrom J, et al. Prognostic value of quality of life scores for time to progression (TTP) and overall survival time (OS) in advanced breast cancer. Eur J Cancer 2003; 39(10): 1370-6.

[18] Debess JE. Cognitive function in relation to treatment of early breast cancer: a population-based longitudinal study: $\mathrm{PhD}$ thesis. [Aarhus]: Faculty of Health Science, University of Aarhus 2008.

[19] Aaronson NK, Ahmedzai S, Bergman B, et al. The European Organization for Research and Treatment of Cancer QLQ-C30: a quality-of-life instrument for use in international clinical trials in oncology. J Natl Cancer Inst 1993; 85(5): 365-76.

[20] Fayers PM, Aaronson NK, Bjordal K, Groenvold M, Curran D, Bottomley A. The EORTC QLQ C-30 Scoring Manual. 3rd ed. Brussels: European Organization for Research and Treatment of Cancer 2001.

[21] Breast. In: Edge SB, Byrd DR, Compton CC, et al., Eds. AJCC Cancer Staging Manual 7th ed. New York, NY: Springer 2010; pp. 347-76.

[22] Klee M, Groenvold M, Machin D. Quality of life of Danish women: population-based norms of the EORTC QLQ-C30. Qual Life Res 1997; 6(1): 27-34.

[23] Sprangers MA, Schwartz CE. Integrating response shift into healthrelated quality of life research: a theoretical model. Soc Sci Med 1999; 48(11): 1507-15.

[24] Osborne RH, Hawkins M, Sprangers MA. Change of perspective: a measurable and desired outcome of chronic disease selfmanagement intervention programs that violates the premise of preintervention/postintervention assessment. Arthritis Rheum 2006; 55(3): 458-65. 